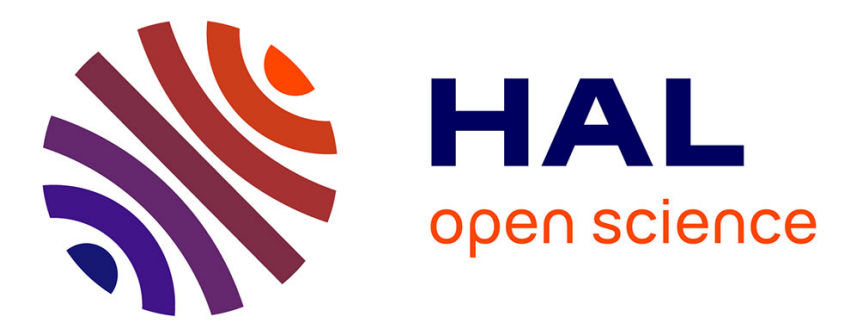

\title{
Proteasome inhibitors to alleviate aberrant IKBKAP mRNA splicing and low IKAP/hELP1 synthesis in familial dysautonomia
}

Mylène Hervé, El Chérif Ibrahim

\section{- To cite this version:}

Mylène Hervé, El Chérif Ibrahim. Proteasome inhibitors to alleviate aberrant IKBKAP mRNA splicing and low IKAP/hELP1 synthesis in familial dysautonomia. Neurobiology of Disease, 2017, 103, pp.113-122. 10.1016/j.nbd.2017.04.009 . hal-01575049

\section{HAL Id: hal-01575049 \\ https://hal-amu.archives-ouvertes.fr/hal-01575049}

Submitted on 17 Aug 2017

HAL is a multi-disciplinary open access archive for the deposit and dissemination of scientific research documents, whether they are published or not. The documents may come from teaching and research institutions in France or abroad, or from public or private research centers.
L'archive ouverte pluridisciplinaire $\mathbf{H A L}$, est destinée au dépôt et à la diffusion de documents scientifiques de niveau recherche, publiés ou non, émanant des établissements d'enseignement et de recherche français ou étrangers, des laboratoires publics ou privés. 


\section{HIGHLIGHTS}

- Proteasome inhibitors increase exon 20 inclusion in IKBKAP mRNA from FD cells.

- Epoxyketones upregulate IKAP/ELP1 protein level in FD cells.

- Carfilzomib modulate expression of microRNAs targeting proteasome subunits.

- Proteasome activity is enhanced in FD cells compared to control cells.

- Combination of already approved drugs may offer novel therapeutic strategy for FD. 


\section{Proteasome inhibitors to alleviate aberrant IKBKAP mRNA}

splicing and low IKAP/hELP1 synthesis in familial dysautonomia

Mylène Hervé ${ }^{1} \&$ El Chérif Ibrahim ${ }^{1,2, *}$

${ }^{1}$ Aix-Marseille Univ, CNRS, CRN2M, 13344 Marseille Cedex 15, France

${ }^{2}$ Aix-Marseille Univ, CNRS, INT, Inst Neurosci Timone, 13385 Marseille Cedex 5, France

*Corresponding author present adress:

El Chérif Ibrahim, Institut de Neurosciences de la Timone (INT), UMR 7289

Campus Santé Timone, 27 Bd Jean Moulin, 13385 Marseille Cedex 5 France

Tel: +33 (0)4 91698956

Email: el-cherif.ibrahim@univ-amu.fr 


\section{ABSTRACT}

FD is a rare neurodegenerative disorder caused by a mutation of the IKBKAP gene, which induces low expression levels of the Elongator subunit IKAP/hELP1 protein. A rational strategy for FD treatment could be to identify drugs increasing IKAP/hELP1 expression levels by blocking protein degradation pathways such as the $26 \mathrm{~S}$ proteasome. Proteasome inhibitors are promising molecules emerging in cancer treatment and could thus constitute an enticing pharmaceutical strategy for FD treatment. Therefore, we tested three proteasome inhibitors on FD human olfactory ecto-mesenchymal stem cells (hOE-MSCs): two approved by the Food and Drug Administration (FDA) and European Medicines Agency (EMA), bortezomib and carfilzomib, as well as epoxomicin. Although all 3 inhibitors demonstrated activity in correcting IKBKAP mRNA aberrant splicing, carfilzomib was superior in enhancing IKAP/hELP1 quantity. Moreover, we observed a synergistic effect of suboptimal doses of carfilzomib on kinetin in improving IKBKAP isoforms ratio and IKAP/hELP1 expression levels allowing to counterbalance carfilzomib toxicity. Finally, we identified several dysregulated miRNAs after carfilzomib treatment that target proteasome-associated mRNAs and determined that IKAP/hELP1 deficiency in FD pathology is correlated to an overactivity of the $26 \mathrm{~S}$ proteasome. Altogether, these results reinforce the rationale for using chemical compounds inhibiting the $26 \mathrm{~S}$ proteasome as an innovative option for FD and a promising therapeutic pathway for many other neurodegenerative diseases.

Keywords: familial dysautonomia, proteasome, carfilzomib, IKBKAP, mRNA splicing, microRNA 


\section{INTRODUCTION}

Familial dysautonomia (FD, also known as Riley Day syndrome or Hereditary Sensory Autonomic Neuropathy type III, MIM 223900) is a neurodevelopmental, autosomal recessive disorder that exclusively affects the Ashkenazi Jewish population with an incidence of 1:3700 live births. This neurodegenerative orphan disease is characterized by a progressive degeneration of fibers from the autonomic and sensory nervous system, which results in clinical manifestations such as pain and temperature sensation loss, gastrointestinal and cardiovascular deficits, dysautonomic crises and complications. No curative treatment is currently available and, despite development of palliative care, about $50 \%$ of patients die before reaching 40 years old (Norcliffe-Kaufmann et al., 2016).

FD is caused by mutations affecting the IKBKAP gene (Anderson et al., 2001; Slaugenhaupt et al., 2001). The most common mutation, which occurs in $99.5 \%$ of FD patients, is a $\mathrm{T}$ to $\mathrm{C}$ transition in position 6 of the donor splice site (5'ss) of intron 20 of the IKBKAP gene (c.2204+6T>C). This mutation leads to an alternative splicing profile of this gene resulting in expression of both IKBKAP WT (exon 20 inclusion) and MU (exon 20 skipping) transcripts, which leads to a decreased synthesis of IKAP/hELP1 protein specifically in nervous system tissues (Cuajungco et al., 2003).

IKAP/hELP1 is a $150-\mathrm{kDa}$ scaffold protein of a well-conserved six-subunit complex, named Elongator (Glatt and Muller, 2013; Hawkes et al., 2002; Xu et al., 2015). At present, the function of IKAP/hElongator is not clearly understood, but it was identified as playing a role in processes such as transcription elongation, $\alpha$-tubulin acetylation, intracellular trafficking, exocytosis and tRNA post-transcriptional modifications (Creppe and Buschbeck, 2011; Karlsborn et al., 2014; Svejstrup, 2007). Moreover, IKAP/hELP1 has an essential function in nervous system development, notably in neuronal cell survival, migration and 
differentiation (Abashidze et al., 2014; Chaverra et al., 2017; Cheishvili et al., 2007; Creppe et al., 2009; Hunnicutt et al., 2012; Jackson et al., 2014; Lefler et al., 2015).

The design of therapeutic strategies is highly dependent on the quality of disease modeling. Cellular models constitute interesting tools but, in the case of an orphan genetic disease like FD, samples are scarce. Therefore, in vitro models derived from easily accessible cells were established. Fibroblasts are readily available from human donors and can be reprogrammed into undifferentiated and self-renewal cells named induced Pluripotent Stem Cells (iPSCs) (Lee et al., 2009). They have been successfully used for modeling several neurological pathologies such as Alzheimer's disease, Parkinson's disease or autism spectrum disorders (Marchetto et al., 2011). However, generation and maintenance of iPSCs remains tedious, and an alternative was found by using human Olfactory Ecto-Mesenchymal Stem Cells (hOE-MSCs). Easily and rapidly harvested from nasal cavity, these native self-renewal cells represent a remarkable model to study genetic diseases by summarizing efficiently molecular deficits of various neuropathologies such as FD (Boone et al., 2010; Mackay-Sim, 2012; Mor et al., 2013; Nguyen et al., 2016).

To better understand pathophysiology of FD, molecular signatures were assessed in various cellular models (Boone et al., 2010; Cheishvili et al., 2011; Close et al., 2006; CohenKupiec et al., 2011; Laguesse et al., 2015; Lee et al., 2009; Zeltner et al., 2016). Genomewide studies revealed a specific transcriptional profile in FD. Among highlighted dysregulated mRNAs, many are related to neurological pathways or cytoskeleton actin regulation (Boone et al., 2012; Cheishvili et al., 2007; Lee et al., 2009). Furthermore, the investigations of transcriptional alterations induced by therapeutic compounds modulating IKBKAP aberrant splicing, such as kinetin and its derivatives (Boone et al., 2012; Lefler et al., 2015; Slaugenhaupt et al., 2004; Yoshida et al., 2015), contributed to a better knowledge of FD pathophysiology. 
The lack of any efficient therapy for FD warrants the testing of various therapeutic approaches. As such, the identification of agents capable to increase the IKAP/hELP1 protein half-life would be of great interest. Protein availability results from the balance between new synthesis and degradation mechanisms. One of the most prevalent cellular pathways for protein degradation is provided by the $26 \mathrm{~S}$ proteasome. This enzymatic multi-subunit complex is the major quality-control operator for newly synthesized proteins (Ravid and Hochstrasser, 2008), and also participates in the regulation of numerous cellular and physiological functions such as cell-cycle progression (King et al., 1996). This complex performs proteolysis of about $20 \%$ of total proteins by recognition of an ubiquitin signal, and then cleavage by trypsin-like, chymotrypsin-like and caspase-like intra-complex activities. Thus, an approach to improve IKAP/hELP1 protein stability could be to block proteasome activity using specific inhibitors. Proteasome inhibitors are structurally diverse small molecules. They can be divided into two large groups based on whether or not they form a covalent bond with the active site threonine and further divided into eight major structural classes (aldehydes, boronates, epoxyketones, $\alpha$-ketoaldehydes, $\beta$-lactones, vinyl sulfones, syrbactins, and bacteria-specific oxatiazol-2-ones) depending on their molecular structures and their specificity with the different enzymatic sites of the proteasome (Kisselev et al., 2012). Numerous natural and synthetic inhibitors are available for in vitro or in vivo laboratory applications. Furthermore, some proteasome inhibitors are being evaluated in clinical trials or are currently used for cancer therapies (Kurtin and Bilotti, 2013). As such, the first generation inhibitor bortezomib (commercial name Velcade, Millennium, Inc. and Johnson and Johnson Pharmaceutical Research and Development) has been approved by the Food and Drug Administration (FDA) since 2003 in refractory multiple myeloma treatment (Scott et al., 2016) and one year later by European Medicines Agency (EMA). The second generation inhibitor carfilzomib (commercial name Kyprolis, Onyx Pharmaceuticals) was 
approved by the FDA in 2012, and three years later by EMA, for multiple myeloma cure on patients who are refractory to immunomodulatory agents and bortezomib (Muchtar et al., 2016). Importantly, carfilzomib is less neurotoxic than bortezomib (Kaplan et al., 2016). Repurposing/repositioning old or recent drugs already approved by national drug regulation authorities (Sardana et al., 2011) offers a pragmatic way to bypass the very lengthy and costly process of drug discovery. As very encouraging results emerged from preclinical testings for another inherited sensory neuropathy, Charcot-Marie-Tooth disease type 1A (Chumakov et al., 2014), there is no reason that such a path should not also be followed for FD.

The proteasome is known to be dynamically associated with microRNAs (miRNAs) (Tsimokha et al., 2014). Indeed, these small non-coding RNAs are posttranscriptional regulators essential for various biological processes such as cellular cycle or neuronal development (Schratt, 2009; Volvert et al., 2012). In addition, miRNAs are also implicated in protein synthesis by acting directly on translation mechanisms, and can modulate protein stability through proteasome interaction. We recently showed that there is a distinct pattern of miRNA expression in FD compared to control cells (Hervé and Ibrahim, 2016). Therefore, targeting the proteasome could be a mechanism to modulate IKAP/hELP1 expression levels. In the context of cancer biology, regulatory loops have recently been described with crosstalk between miRNAs, proteasome machinery and tumor suppressor genes (Zhang et al., 2015). Interestingly, IKAP/hELP1 deficiency is related to expression variation of pro-apoptotic p53dependant genes (Cornez et al., 2008), suggesting that there could be an alteration of the proteasome activity in FD. Indeed, some papers have highlighted ubiquitin-proteasome system impairment in neurodegenerative pathologies such as Parkinson's disease and Alzheimer's disease (Dennissen et al., 2012).

In the present work, our strategy was to test alternative clinical uses of FDA-approved drugs. First, we explored the consequences of incubating FD hOE-MSCs with the first 
generation proteasome inhibitor bortezomib on the alternative splicing profile of the IKBKAP mRNA and IKAP/hELP1 protein expression levels. Next, we tested carfilzomib, a secondgeneration proteasome inhibitor, less neurotoxic than bortezomib, as well as another epoxyketone classically used for in vitro studies, epoxomicin. All three inhibitors favorably modified the IKBKAP transcript profile. In particular, carfilzomib increased the IKAP/hELP1 protein expression level in FD cells. Moreover, we achieved an additive effect of suboptimal doses of carfilzomib combined with kinetin on IKBKAP mRNA and IKAP/hELP1 protein expression levels. Then, we identified dysregulated miRNAs under optimal doses of carfilzomib. Finally, after highlighting validated interactions between dysregulated miRNAs and proteasome subunits, we analyzed the intrinsic activity of proteasome in both control and FD hOE-MSCs and identified an overactive proteasome in FD cells. 


\section{MATERIAL AND METHODS}

\section{Cell culture of hOE-MSCs and drug application}

hOE-MSCs derived from 4 FD patients (FD1, FD2, FD3, FD4) and 4 healthy controls (C1, C2, C3, C4), previously described(Boone et al., 2012; Boone et al., 2010), were cultured in DMEM/HAM'S F12 medium (Life Technologies, Carlsbad, CA) supplemented with $10 \%$ Fetal Bovine Serum (FBS) (PAA, Pasching, Austria) at $37^{\circ} \mathrm{C}$ in an atmosphere at $5 \% \mathrm{CO}_{2}$. Drug solutions were dissolved in culture medium at the indicated concentrations: bortezomib (UBPBio, Aurora, CO), epoxomicin (Sigma-Aldrich), carfilzomib (UBPBio) and kinetin (Sigma-Aldrich). Adherent cells were detached after washing with phosphate-buffered saline (PBS) (Life Technologies, Gibco, Grand Island, NY) and incubation with trypsin-EDTA (0.05\% Trypsin, $0.5 \mathrm{mM}$ EDTA, Life Technologies, Gibco) during $5 \mathrm{~min}$ at $37^{\circ} \mathrm{C}$. Cell pellet was collected after 5 min of centrifugation at $300 \mathrm{~g}$.

\section{Measurement of cell viability with crystal violet assay}

Two control (C1, C3) and two FD (FD2, FD4) cultures of hOE-MSCs were treated with suitable concentrations of proteasome inhibitors for $48 \mathrm{~h}$ and then washed once with PBS. Cells were then incubated with $0.2 \%$ crystal violet solution supplemented with $2 \%$ ethanol for $10 \mathrm{~min}$ at room temperature. After three washes with deionized $\mathrm{H}_{2} \mathrm{O}$, cells were dried overnight protected from light, and then stain was solubilized by treatment with $1 \%$ SDS with agitation until uniform in color. Absorbance at $530 \mathrm{~nm}$ was read in 3-5 min post staining. Each concentration treatment was performed in duplicate. The background signal was subtracted from wells containing $1 \%$ SDS only.

\section{Total RNA isolation}


Total RNA was purified from cell pellets using the mirVana miRNA isolation kit (Life Technologies, Ambion, Austin, TX) according to the manufacturer's recommendations, and subsequently submitted to DNase treatment (DNA-free kit, Life Technologies, Ambion). RNA concentration was measured using a nanodrop ND-1000 spectrophotometer (Thermo Fisher Scientific, Waltham, MA).

\section{Quantification of mRNA expression level}

$1 \mu \mathrm{g}$ of total RNA from each sample was reverse transcribed using the High-Capacity cDNA Reverse Transcription kit (Life Technologies, Applied Biosystems, Foster City, CA). 50 ng of the resulting cDNA were used to perform real-time PCR reactions for each assay, using the TaqMan Universal PCR Master Mix II with no UNG (Life Technologies, Applied Biosystems), on an ABI PRISM 7900HT thermocycler under the following conditions: 10 min at $95^{\circ} \mathrm{C}, 50$ cycles of $15 \mathrm{sec}$ at $95^{\circ} \mathrm{C}$ and $1 \mathrm{~min}$ at $60^{\circ} \mathrm{C}$. Each reaction was performed in duplicate. Primers hELP1 ex19F, hELP1 ex20-21R and probe P-WTELP1 ex20R were used to detect IKBKAP transcripts containing exon 20, and primers hELP1 ex19-21F, hELP1 ex2122R and probe P-MUELP1 ex $21 \mathrm{~F}$ were involved in detection of IKBKAP transcripts deleted of exon 20 (Boone et al., 2010) (Additional File 1). Primers/TaqMan probe assays purchased from Applied Biosystems were used to determine the level of expression of DDIT3 (Hs00358796_g1) as well as the reference gene, WDR59 (Hs00226608_m1). Raw Ct values were obtained with manual baseline settings on the RQ Manager software, and then the relative expression level of each mRNA was quantified by using the $2^{-\Delta \Delta \mathrm{Ct}}$ method (Livak and Schmittgen, 2001).

\section{Western immunoblotting}

Cell pellets were resuspended in 1 X SDS loading buffer at $6 \times 10^{3}$ cells $/ \mu \mathrm{L}$, and then samples were heated at $95^{\circ} \mathrm{C}$ during $5 \mathrm{~min}$. Thereafter, total protein from $10 \mu 1$ of cell lysates were 
separated on $10 \%$ SDS-polyacrylamide gel electrophoresis and transferred to a PVDF membrane (Thermo Fisher Scientific, Rockford, IL). Membranes were blocked with 5\% Milk in PBS supplemented with 0.1\% Tween-20 (Sigma-Aldrich, St. Louis, MO) buffer for 1h at room temperature, and were then subsequently probed at $4{ }^{\circ} \mathrm{C}$ overnight with mouse monoclonal anti-IKAP/hELP1 antibody diluted at 1:2000 (BD Biosciences, Franklin Lakes, NJ) in $2.5 \%$ Milk in PBST, followed by incubation with mouse secondary antibodies at 1:3500 (Sigma-Aldrich). As a control of equal protein loading, membranes were also probed with mouse anti- $\beta$-actin primary antibody used at 1:10000 (Sigma-Aldrich). Stained proteins were revealed by chemiluminescent detection using ECL detection kit (Thermo Fisher Scientific, Rockford, IL) on a G:BOX Chemi XT4 device (Syngene, Cambridge, UK).

\section{MiRNA quantification by microfluidics arrays}

$120 \mathrm{ng}$ of total RNA from four healthy control (C1, C2, C3 and C4) and four FD patients hOE-MSCs (FD1, FD2, FD3 and FD4), treated with or without carfilzomib, were reverse transcribed into cDNA using the TaqMan MicroRNA Reverse Transcription kit (Life Technologies, Applied Biosystems) in combination with the stem-loop Megaplex RT primers, Human Pool A v2.1 (Life Technologies, Applied Biosystems), according to the manufacturer's recommendation. 16 ng of the resulting cDNA was combined with TaqMan Universal PCR Master Mix II with no UNG and $100 \mu \mathrm{L}$ of mix was loaded into each port of the 384 wells TaqMan Human MicroRNA Array A v2.0 (Life Technologies, Applied Biosystems). PCR reactions were run on an ABI PRISM 7900HT thermocycler under the following conditions: $2 \mathrm{~min}$ at $50^{\circ} \mathrm{C}, 10 \mathrm{~min}$ at $95^{\circ} \mathrm{C}, 40$ cycles of $15 \mathrm{sec}$ at $95^{\circ} \mathrm{C}$ and $1 \mathrm{~min}$ at $60^{\circ} \mathrm{C}$. Raw Ct values were calculated using the RQ Manager software with manual baseline settings for each miRNA. Only those miRNAs detectable $(\mathrm{Ct}<35$ in $>25 \%$ of the 16 hOEMSCs samples) were subsequently analyzed. The relative expression level of each miRNA was quantified as $2^{-\Delta \Delta \mathrm{Ct}}$, and therefore normalized based on the expression level of a reference 
miRNA, exhibiting a stable level of expression among all samples and selected with DataAssist and NormFinder softwares, and a calibrator sample (defined by the mean Ct of the control samples without drug). In order to normalize the miRNAs with reference miRNA exhibiting a proximal expression level, 5 windows of expression levels $(\mathrm{Ct}<24 ; 24<\mathrm{Ct}<$ $27 ; 27<\mathrm{Ct}<30 ; 30<\mathrm{Ct}<33 ; \mathrm{Ct}>33$ ) were defined in which a specific reference miRNA was selected.

\section{In silico target prediction}

Signaling pathways linked to dysregulated miRNAs were determined using the DIANAmirPath v3.0 web server that utilizes the DIANA-TarBase v7.0 validated database and predicted miRNA targets (in CDS or 3'-UTR regions) provided by the microT-CDS v5.0 and TargetScan algorithm (Vlachos et al., 2015b). The software was used in the union genes mode, False Discovery Rate (FDR) was enabled and various thresholds were set at 0.05 for the $P$-value, 0.8 for the microT and 0.1 for the TargetScan Conservation Score. For each dysregulated miRNA, validated mRNA targets were provided by the DIANA-TarBase v7.0 (Vlachos et al., 2015a), the miRTarBase (Chou et al., 2016), and the miRWalk2.0 (Dweep and Gretz, 2015) databases. Identified targets were compared to a list of 190 ubiquitin/proteasome-related genes (Additional File 2) obtained from the ubiquitin proteasome dependent proteolysis pathway from the pathcards unification database (https://pathcards.genecards.org/) (Belinky et al., 2015), as well as using the Hugo Gene Nomenclature Committee (HGNC) website (http://www.genenames.org/) in the categories "proteasome (PSM)", "ubiquitin-like modifier activating enzymes (UBA)”, "ubiquilin family (UBQLN)", "ubiquitin C-terminal hydrolases (UCHL)", "ubiquitin conjugating enzymes E2 (UBE2)", and "ubiquitin specific peptidases (USP)". Common mRNAs were retained in order to generate miRNA:mRNA couples. For each dysregulated miRNA, in silico predicted targets were also identified using the combinatorial miRNA target module of miRWalk2.0 by 
retaining only the target candidates given by the union of at least 7 out of 10 algorithms (miRWalk, miRDB, PITA, MicroT4, miRMap, RNA22, miRanda, RNAhybrid, PICTAR2 and Targetscan).

\section{Proteasome activity}

Proteasome activity was measured on two control (C1 and C3) and two FD (FD2 and FD4) hOE-MSCs using the Proteasome Activity Fluorometric Assay Kit (BioVision, Milpitas, CA). Briefly, adherent cells were detached, counted then resuspended in 0.5\% NP-40 in PBS solution at a concentration of $15 \times 10^{4}$ cells in $100 \mu \mathrm{L}$. In a white opaque 96-wells plate, $20 \mu \mathrm{L}$ of cell suspension (30 000 cells) was mixed with $80 \mu \mathrm{L}$ of proteasome assay buffer. Each cell culture was tested in triplicate. Kinetics of fluorescence development at Excitation/Emission = 350/440 nm was obtained on a SpectraMax M2/ Microplate Reader (Molecular Devices, Sunnyvale, CA), with one measurement every 5 minutes over 90 minutes. The signal generated by proteasome activity was calculated as the variation in Relative Fluorescence Unit $(\triangle \mathrm{RFU})=\mathrm{RFU}$ TIME $2-\mathrm{RFU}$ TIME 1 , with RFU corresponding to the signal intensity measured twice along 30 minutes in the same wells.

\section{Statistical analysis}

Differentially expressed miRNAs in each group comparison were arbitrarily selected as miRNAs presenting a FC superior to 1.3 , since no scientific consensus was established for FC threshold, then coupled with statistical analysis using paired mode of the parametric Student's t-test to compare the FC between treated and untreated cells using the IBM SPSS Statistics v20 software. The same statistical test was used to validate individual dysregulation of selected miRNAs as well as IKBKAP mRNA expression variations. The threshold $P$-value was set to 0.05 . 


\section{RESULTS}

\section{Optimal concentration range for bortezomib treatment on hOE-MSCs}

Proteasome inhibitors had not previously been tested on hOE-MSCs. Therefore, to define adequate testing dose, cell sensitivity to increasing concentrations of proteasome inhibitors was monitored using the crystal violet assay. Two control and two FD hOE-MSC cultures were treated for $48 \mathrm{~h}$ with bortezomib concentration ranging from 1 to $1000 \mathrm{nM}$ (Fig. 1A). The drug induced very similar toxicity toward control and FD hOE-MSCs with a median lethal dose (LD50) close to $10-15 \mathrm{nM}$. To further study the effect of bortezomib on RNA and protein expression, we focused on concentrations preserving at least a third of cells alive ( $\leq$ $30 \mathrm{nM})$. At those concentrations, the pharmacologic activity was validated by assessing expression level variations of DDIT3, a gene known to be modulated by proteasome inhibitors (Fu et al., 2008; Mimura et al., 2014; Sado et al., 2009) (Additional File 3A).

\section{Bortezomib increases exon 20 inclusion in IKBKAP mRNA but not IKAP/hELP1 protein expression}

Because the proteasome is well connected to different gene expression machines(Komili and Silver, 2008), we first tested whether proteasome inhibitors could perturb IKBKAP mRNA alternative splicing. Indeed, application for $48 \mathrm{~h}$ of bortezomib on FD4 hOE-MSCs boosted the WT:MU ratio up to about 6 times with a plateau observed between 15 and $30 \mathrm{nM}$ (Fig. 1B). Such improvement of IKBKAP exon 20 inclusion was not correlated to an IKAP/hELP1 accumulation at the protein level (Fig. 1C).

We next investigated whether it could be possible to moderate the side effects of bortezomib by combining it to kinetin, the most active compound known to correct IKBKAP mRNA aberrant splicing and increase IKAP/hELP1 expression. With $10 \mathrm{nM}$ of bortezomib (Fig. 1A 
and $1 \mathrm{~B}$, arrow) and $25 \mu \mathrm{M}$ of kinetin (Additional File 4, arrow). we observed a synergistic effect (40 times enhancement) on IKBKAP exon 20 inclusion compared to the use of drugs as a single agent which upregulated WT:MU ratio about 7 times (Fig.1D). Nevertheless, a much milder effect was observed on IKAP/hELP1 protein expression levels (Fig. 1E). For a more complete improvement of genetic expression, we next explored the use of another family of proteasome inhibitors, epoxyketones.

\section{Optimal concentration range for epoxomicin on hOE-MSCs}

Epoxomicin is a widely used research tool in biochemistry and cell biology that produced substantial cell mortality as assessed by the crystal violet assay (Fig. 2A). However, FD cells were not affected more than control cells. After $48 \mathrm{~h}$ of epoxomicin application, cell mortality reached 50\% with $30 \mathrm{nM}$ epoxomicin. We selected $30 \mathrm{nM}$ as an upper limit for examining the effect of epoxomicin on RNA and protein expression. hOE-MSCs were responsive to the pharmacologic activity of epoxomicin by showing elevation of DDIT3 gene expression (Additional File 3B).

Epoxomicin increases exon 20 inclusion in IKBKAP $\mathrm{mRNA}$ and IKAP/hELP1 protein expression level alone or combined to kinetin

Increasing concentrations of epoxomicin induced a sustained upregulation in the ratio of WT:MU transcripts over 20 times at $30 \mathrm{nM}$ (Fig. 2B). In accordance with transcriptional results, up to 13-fold enhancement of IKAP/hELP1 detection was obtained with epoxomicin (Fig. 2C). To strengthen such an effect and to reduce cytotoxicity, we combined suboptimal doses of epoxomicin with kinetin. Thus, we retained concentration of $15 \mathrm{nM}$ for epoxomicin (Fig. 2A and 2B, arrows). Interestingly, both at the splicing (Fig. 2D) and at the translational level (Fig. 2E), co-incubation of epoxomicin and kinetin provided an additive effect compared to the use of each drug alone. 
To progress in our hypothesis to propose proteasome inhibitors as potential therapeutic compounds in FD, we decided to study the effect of another proteasome inhibitor belonging to the same chemical family as epoxomicin and approved by the FDA, carfilzomib, an agent currently used for multiple myeloma treatment.

\section{Strong impact of carfilzomib alone or combined to other drugs on IKBKAP alternative splicing and IKAP/hELP1 expression level}

Increasing doses of carfilzomib incubated for $48 \mathrm{~h}$ demonstrated similar decrease in viability on two control and two FD hOE-MSCs, with $60 \mathrm{nM}$ resulting in about $50 \%$ of cell mortality (Fig. 3A). Thereafter, we tested increasing concentrations of carfilzomib (5 to $125 \mathrm{nM}$ ) on FD4 cells for $48 \mathrm{~h}$. Starting at $15 \mathrm{nM}$, the WT:MU ratio of IKBKAP transcripts really increased with a maximal effect of 5-8 fold enhancement from 30 to $125 \mathrm{nM}$ (Fig. 3B). In accordance with transcriptional data, a maximal detection of IKAP/hELP1 protein was observed at $125 \mathrm{nM}$ of carfilzomib (Fig. 3C). We also explored the effect on cell viability of lowering proteasome inhibitor without losing IKAP/hELP1 enhancement by combining carfilzomib and kinetin. As shown on Figure 3D and 3E, lower doses of carfilzomib (30 nM, Fig. 3A and 3B, arrows) and kinetin (25 $\mu \mathrm{M}$, Additional File 4) produced synergistic and additive effects on WT:MU ratio of IKBKAP transcripts and IKAP/hELP1 protein pattern, respectively.

Next, we decided to explore the link between the proteasome inhibitors and the modified miRNA pattern of expression. Indeed, miRNAs are small non-coding RNAs known to play a role in various neurodegenerative pathologies and appearing as innovative potential therapeutic target (Basak et al., 2016). Moreover, we previously demonstrated existence of a specific miRNA signature in FD (Hervé and Ibrahim, 2016), leading to the hypothesis that a therapeutic effect of some pharmacological agents could be mediated by miRNAs. We thus 
profiled dysregulated miRNAs under carfilzomib treatment on healthy control and FD patient cells.

\section{Impact of carfilzomib on IKBKAP transcripts in control and FD hOE-MSCs cultures}

Before studying miRNA variations, we firstly validated the impact of carfilzomib treatment for $24 \mathrm{~h}$ on IKBKAP mRNA pattern in four control hOE-MSCs $(\mathrm{C} 1, \mathrm{C} 2, \mathrm{C} 3$ and $\mathrm{C} 4)$ and four FD hOE-MSCs (FD1, FD2, FD3 and FD4). No significant modulation of IKBKAP WT transcript was obtained after carfilzomib treatment in healthy control hOE-MSCs (Additional File 5). Conversely, the level of IKBKAP WT transcript more than doubled in FD hOE-MSCs in the presence of carfilzomib, concomitant to a decrease of the IKBKAP MU transcript (Additional File 5).

\section{Carfilzomib induces a specific miRNA signature in control and FD cells}

The same 16 samples tested for IKBKAP mRNA expression were profiled by the simultaneous amplification of 381 small RNAs, including 373 human miRNAs (Additional File 6). The raw data indicated that around $80 \%$ of these small RNAs were expressed (mean $\mathrm{Ct}<40$ ) in our cells (Additional File 7). However, we only conserved miRNAs presenting $\mathrm{Ct}$ $<35$ in $>25 \%$ of samples for subsequent analyses. These 185 analyzable small RNAs were clustered into five classes according to their level of expression (Additional File 8).

Next, we compared untreated condition to carfilzomib treatment and identified a list of miRNAs after an arbitrary setting of $\mathrm{FC} \geq 1.3$ and challenging the statistical significance of the results with paired Student's t-test. Five miRNAs were found dysregulated under carfilzomib application on control hOE-MSCs and fourteen in FD hOE-MSCs (Table 1). Interestingly, miR-126-3p, overexpressed by carfilzomib in both control and FD cells (Table 1), had previously been shown to be underexpressed in FD compared to control state (Hervé and Ibrahim, 2016). Moreover, miR-365a-3p and miR-636, dysregulated in FD pathology 
(Hervé and Ibrahim, 2016), were both modulated in the reverse way by carfilzomib treatment on FD cells (Table 1).

\section{Proteasome subunits as validated targets of dysregulated miRNAs in FD hOE-MSCs under carfilzomib application}

To better understand implication of the dysregulated miRNAs under carfilzomib treatment in proteasome degradation systems, we determined the biological pathways related to the 17 miRNAs differentially expressed between carfilzomib-untreated and -treated hOE-MSCs with carfilzomib by using various databases and algorithms from the DIANA-mirPath v3.0 software (Vlachos et al., 2015b). As expected for the activity of a proteasome inhibitor, significantly altered biological processes in both control and FD hOE-MSCs by carfilzomib treatment included intracellular recycling processes such as ubiquitin-mediated proteolysis (Additional File 9). Other biological processes involved in carfilzomib response concerned neuronal, developmental processes as well as intracellular signaling, metabolism, carcinogenesis and infectious diseases (Additional File 9). Of note, transcription-translationsplicing processes were specifically altered in FD hOE-MSCs (Additional File 9).

We also conducted an in silico search of validated mRNA targets for each dysregulated miRNA. We specifically focused our exploration on targets related to the ubiquitin/proteasome activities (Additional File 2), and highlighted that several proteasomerelated mRNAs were targeted by carfilzomib-induced dysregulated miRNAs (Table 2A, Additional File 10). Furthermore, we investigated whether the nine miRNAs previously selected among dysregulated miRNAs in FD pathology (Hervé and Ibrahim, 2016) (miR-1263p, miR-137, miR-146a-5p, miR-203a-3p, miR-324-3p, miR-339-5p, miR-345-5p, miR-376c3p, miR-541-3p) could also target proteasome mRNAs. Using the same method, we demonstrated that mRNAs of the proteasome biological pathway were targeted by these 
miRNAs, with five out of nine directly targeting proteasome subunits, suggesting that proteasome activity could be altered in the physiopathology of FD (Table 2B, Additional File 11).

\section{Overactivity of $26 \mathrm{~S}$ proteasome in FD compared to control hOE-MSCs.}

MiRNAs dysregulated in FD pathology and after carfilzomib treatment are associated with proteasome-related mRNA targets. This suggests a proteasome malfunction and we surmised that a low expression level of IKAP/hELP1 protein could be accentuated by proteasome overactivity in FD patients. We measured proteasome activity in two healthy control (C1 and C3) and two FD hOE-MSCs (FD2 and FD4) by evaluating chymotrypsin-like activity of the $26 \mathrm{~S}$ proteasome using a fluorescence assay. Of note, we observed an increase of $28 \%$ of the proteasome activity in FD compared to control hOE-MSCs (Figure 4). 


\section{DISCUSSION}

FD is a fatal rare disease caused by a mutation in the IKBKAP gene, which induces a progressive degenerative loss of the autonomic and sensory nervous system. No cure is available and there is a high unmet need in advancing therapeutic strategies for FD. However, development of new pharmaceutical compounds is a long and expensive process, complicated by the orphan nature of the FD pathology. A rational therapeutic strategy thus consists of using pharmaceuticals already approved by pharmaceutical administrations and available on the market. Ideally, the drug would be able to increase expression levels of the deficient protein IKAP/hELP1. In theory, this could occur by inhibiting protein degradation pathways to lengthen the half-life of the protein. To achieve this goal, two main well-defined protein degradation pathways can be targeted, autophagy mechanisms and/or $26 \mathrm{~S}$ proteasome. Interestingly, several agents targeting the $26 \mathrm{~S}$ proteasome are already available in cancer treatment and new drugs targeting the proteasome are continuously being discovered (Mitsiades, 2015). Moreover, some of these proteasome inhibitors appeared to be successfully derived to treat other pathologies such as muscular dystrophy (Azakir et al., 2014) and could constitute an attractive strategy for FD.

In this study, we hypothesized that proteasome inhibition would improve IKAP/hELP1 expression levels. We tested first and second generation inhibitors used in the clinics for cancer treatment (bortezomib and carfilzomib) as well as a classical inhibitor used for in vitro studies (epoxomicin) on FD hOE-MSCs. In agreement with our hypothesis, both epoxyketones, epoxomicin and carfilzomib, induced a significant increase of IKAP/hELP1 protein quantity. Interestingly, we highlighted in parallel that all proteasome inhibitors modulate aberrant alternative splicing of the IKBKAP gene. Such an observation was not anticipated but the proteasome is one of the many interconnected protein machinery, such as 
chromatin remodelers, transcription factors and the RNA polymerase, that together play important roles in regulatory processes (Komili and Silver, 2008). Our observation can also be related to previous studies that indicated an interconnection between $26 \mathrm{~S}$ proteasome and the spliceosome machinery (Bellare et al., 2008; Bieler et al., 2012), including splicing factors such as SR proteins and hnRNPs (Koumbadinga et al., 2015; Moulton et al., 2014). To our knowledge, this is the first study highlighting modulation of IKBKAP mRNA alternative splicing and IKAP/hELP1 expression levels by proteasome inhibitors in FD pathology.

We chose to focus on carfilzomib, because the compound has been shown to be less neurotoxic than bortezomib (Arastu-Kapur et al., 2011; Kaplan et al., 2016). This FDAapproved compound, if further confirmed to provide curative benefits in vitro and ex vivo, may be readily available to FD patients, without enduring lengthy and costly trials assessing toxicity and side effects. However, because sensitivity of cells may be different from patients, a further difficulty will be to define the adequate dose in vivo to reproduce in vitro effects. Carfilzomib, used at a similar concentration range as in previous studies (Lamothe et al., 2015), improves IKBKAP mRNA aberrant splicing and IKAP/hELP1 expression levels but is relatively cytotoxic on hOE-MSCs. To overcome such an issue, a strategy is to combine low doses of carfilzomib with other molecules correcting IKBKAP mRNA splicing such as kinetin or RECTAS (Slaugenhaupt et al., 2004; Yoshida et al., 2015). When we tested kinetin, we revealed a synergic activity of this molecule at a suboptimal dose with either epoxomicin or carfilzomib on IKBKAP mRNA aberrant splicing profile and IKAP/hELP1 protein level. Remarkably, an antioxidant flavonoid named epigallocatechin gallate (EGCG), which is the most abundant and bioactive polyphenol in green tea, has been identified by the Rubin group as capable of correcting IKBKAP mRNA aberrant splicing by decreasing hnRNP A2/B1 expression level (Anderson et al., 2012; Anderson et al., 2003a). Although its efficacy in FD remains controversial (Lee et al., 2009), its anticancer properties have been in part related to 
proteasome inhibition (Chen et al., 2011; Modernelli et al., 2015). Therefore, a rational approach to reduce carfilzomib toxicity while maintaining splicing profile improvement and proteasome blocking could be to combine this drug with EGCG or with tocotrienols, members of the vitamin E family, that have been shown to upregulate IKBKAP transcription and to provide clinical benefit to FD patients (Anderson et al., 2003b; Cheishvili et al., 2016).

Proteasome inhibitors are pharmacological agents mainly used to kill tumor cells but also able to induce toxicity towards healthy cells. Indeed, protein degradation is a complex process involving interconnection of several mechanisms including the $26 \mathrm{~S}$ proteasome, autophagy and unfolded protein response (UPR) pathways. Thus, any therapeutic strategy must ensure that beneficial modulation of one degradation process is not deleterious to the others. Notably, a link was recently established between the weak activity of the Elongator complex in ELP3 conditional knockout mice and UPR-pathway destabilization, correlating with an inability to produce neurons during corticogenesis (Laguesse et al., 2015). We thus surmised that IKAP/hELP1 deficiency could induce proteasome alterations and we demonstrated that Elongator dysfunction in FD disturbs proteasome activity. Mechanistically, we suggested in a previous work that FD patients could develop an adaptation mechanism to cope with Elongator deficiencies. Indeed, we underscored that FD hOE-MSCs present a specific miRNA profile able to influence mRNAs expression pattern, such as the NOVA1 splicing factor (Hervé and Ibrahim, 2016). Therefore, we propose that the same mechanism of action could impact on protein degradation processes. We highlighted several miRNAs significantly dysregulated after carfilzomib treatment and targeting proteasome-associated mRNAs. We found two miRNAs, miR-126-3p and miR-636, that were commonly dysregulated in control and FD hOE-MSCs and could thus help to define the mechanisms of carfilzomib action on IKBKAP. We also revealed that miR-137, previously shown to be dysregulated in FD pathology(Hervé and Ibrahim, 2016), is affected by carfilzomib treatment 
in FD cells. Of note, miR-137 is known to play a role on nervous system development by acting, among other processes, on the regulation of neural stem cells proliferation (Volvert et al., 2012). Future experiments will aim to investigate whether restoration of normal expression of miR-137, miR-126-3p and miR-636 directly dampen abnormal proteasome activity. 


\section{CONCLUSIONS}

This work highlights positive effects of proteasome inhibitors on IKBKAP mRNA aberrant splicing and IKAP/hELP1 expression level. Some of these inhibitors are already approved for clinical treatment of cancer and could serve in combination with other compounds used in cosmetics or nutritional supplements as an alternative strategy for an FD cure. Ideally, it would be interesting to use of FD neuronal cells to validate these drugs and to assess their action on animal models to analyze IKAP/hELP1 expression variation in relation to improvement of symptoms (Morini et al., 2016; Naftelberg et al., 2016; Ueki et al., 2016). Ultimately, this strategy of proteasome inhibition developed for a rare disease can be adapted to various neurodegenerative diseases. 


\section{ACKNOWLEDGMENTS}

This work is dedicated to the memory of David Brenner who played a leading role in supporting researchers working on FD worldwide. We wish to thank the patients and their families for their support to this study. We thank Felicia Axelrod for her invaluable help to make possible our investigations. We also thank Julie-Ann Gavigan and Jeanne Hsu for editing the manuscript. This work was supported by research grants from Association Française contre les Myopathies and the Dysautonomia Foundation. MH was supported by a PhD fellowship from the Ministère de l'Enseignement Supérieur et de la Recherche (MESR). 


\section{REFERENCES}

Abashidze, A., et al., 2014. Involvement of IKAP in peripheral target innervation and in specific JNK and NGF signaling in developing PNS neurons. PLoS One. 9, e113428.

Anderson, S. L., et al., 2001. Familial dysautonomia is caused by mutations of the IKAP gene. Am. J. Hum. Genet. 68, 753-8.

Anderson, S. L., et al., 2012. Nutraceutical-mediated restoration of wild-type levels of IKBKAP-encoded IKAP protein in familial dysautonomia-derived cells. Mol. Nutr. Food Res. 56, 570-9.

Anderson, S. L., et al., 2003a. EGCG corrects aberrant splicing of IKAP mRNA in cells from patients with familial dysautonomia. Biochem. Biophys. Res. Commun. 310, 627-33.

Anderson, S. L., et al., 2003b. Tocotrienols induce IKBKAP expression: a possible therapy for familial dysautonomia. Biochem. Biophys. Res. Commun. 306, 303-9.

Arastu-Kapur, S., et al., 2011. Nonproteasomal targets of the proteasome inhibitors bortezomib and carfilzomib: a link to clinical adverse events. Clin. Cancer Res. 17, 2734-43.

Azakir, B. A., et al., 2014. Proteasome inhibitors increase missense mutated dysferlin in patients with muscular dystrophy. Sci. Transl. Med. 6, 250ra112.

Basak, I., et al., 2016. microRNAs as neuroregulators, biomarkers and therapeutic agents in neurodegenerative diseases. Cell. Mol. Life Sci. 73, 811-27.

Belinky, F., et al., 2015. PathCards: multi-source consolidation of human biological pathways. Database (Oxford). 2015.

Bellare, P., et al., 2008. A role for ubiquitin in the spliceosome assembly pathway. Nat. Struct. Mol. Biol. 15, 444-51.

Bieler, S., et al., 2012. Low dose proteasome inhibition affects alternative splicing. J. Proteome Res. 11, 3947-54. 
Boone, N., et al., 2012. Genome-wide analysis of familial dysautonomia and kinetin target genes with patient olfactory ecto-mesenchymal stem cells. Hum. Mutat. 33, 530-40.

Boone, N., et al., 2010. Olfactory stem cells, a new cellular model for studying molecular mechanisms underlying familial dysautonomia. PLoS One. 5, e15590.

Chaverra, M., et al., 2017. The Familial Dysautonomia disease gene, Ikbkap/Elp1, is required in the developing and adult central nervous system. Dis. Model Mech.

Cheishvili, D., et al., 2011. IKAP/Elp1 involvement in cytoskeleton regulation and implication for familial dysautonomia. Hum. Mol. Genet. 20, 1585-94.

Cheishvili, D., et al., 2016. Tocotrienol Treatment in Familial Dysautonomia: Open-Label Pilot Study. J. Mol. Neurosci. 59, 382-91.

Cheishvili, D., et al., 2007. IKAP/hELP1 deficiency in the cerebrum of familial dysautonomia patients results in down regulation of genes involved in oligodendrocyte differentiation and in myelination. Hum. Mol. Genet. 16, 2097-104.

Chen, D., et al., 2011. EGCG, green tea polyphenols and their synthetic analogs and prodrugs for human cancer prevention and treatment. Adv. Clin. Chem. 53, 155-77.

Chou, C. H., et al., 2016. miRTarBase 2016: updates to the experimentally validated miRNAtarget interactions database. Nucleic Acids Res. 44, D239-47.

Chumakov, I., et al., 2014. Polytherapy with a combination of three repurposed drugs (PXT3003) down-regulates Pmp22 over-expression and improves myelination, axonal and functional parameters in models of CMT1A neuropathy. Orphanet J. Rare Dis. 9, 201.

Close, P., et al., 2006. Transcription impairment and cell migration defects in elongatordepleted cells: implication for familial dysautonomia. Mol. Cell. 22, 521-31. 
Cohen-Kupiec, R., et al., 2011. Effects of IKAP/hELP1 deficiency on gene expression in differentiating neuroblastoma cells: implications for familial dysautonomia. PLoS One. 6, e19147.

Cornez, I., et al., 2008. Deregulated expression of pro-survival and pro-apoptotic p53dependent genes upon Elongator deficiency in colon cancer cells. Biochem. Pharmacol. 75, 2122-34.

Creppe, C., Buschbeck, M., 2011. Elongator: an ancestral complex driving transcription and migration through protein acetylation. J. Biomed. Biotechnol. 2011, 924898.

Creppe, C., et al., 2009. Elongator controls the migration and differentiation of cortical neurons through acetylation of alpha-tubulin. Cell. 136, 551-64.

Cuajungco, M. P., et al., 2003. Tissue-specific reduction in splicing efficiency of IKBKAP due to the major mutation associated with familial dysautonomia. Am. J. Hum. Genet. $72,749-58$.

Dennissen, F. J., et al., 2012. The ubiquitin proteasome system in neurodegenerative diseases: culprit, accomplice or victim? Prog. Neurobiol. 96, 190-207.

Dweep, H., Gretz, N., 2015. miRWalk2.0: a comprehensive atlas of microRNA-target interactions. Nat. Methods. 12, 697.

Fu, H. Y., et al., 2008. Overexpression of endoplasmic reticulum-resident chaperone attenuates cardiomyocyte death induced by proteasome inhibition. Cardiovasc. Res. 79, 600-10.

Glatt, S., Muller, C. W., 2013. Structural insights into Elongator function. Curr. Opin. Struct. Biol. 23, 235-42.

Hawkes, N. A., et al., 2002. Purification and characterization of the human elongator complex. J. Biol. Chem. 277, 3047-52. 
Hervé, M., Ibrahim, E. C., 2016. MicroRNA screening identifies a link between NOVA1 expression and a low level of IKAP in familial dysautonomia. Dis. Model Mech. 9, 899-909.

Hunnicutt, B. J., et al., 2012. IKAP/Elp1 is required in vivo for neurogenesis and neuronal survival, but not for neural crest migration. PLoS One. 7, e32050.

Jackson, M. Z., et al., 2014. A neuron autonomous role for the familial dysautonomia gene ELP1 in sympathetic and sensory target tissue innervation. Development. 141, 245261.

Kaplan, G. S., et al., 2016. Proteasome inhibitors in cancer therapy: Treatment regimen and peripheral neuropathy as a side effect. Free Radic. Biol. Med. 103, 1-13.

Karlsborn, T., et al., 2014. Elongator, a conserved complex required for wobble uridine modifications in eukaryotes. RNA Biol. 11, 1519-28.

King, R. W., et al., 1996. How proteolysis drives the cell cycle. Science. 274, 1652-9.

Kisselev, A. F., et al., 2012. Proteasome inhibitors: an expanding army attacking a unique target. Chem. Biol. 19, 99-115.

Komili, S., Silver, P. A., 2008. Coupling and coordination in gene expression processes: a systems biology view. Nat. Rev. Genet. 9, 38-48.

Koumbadinga, G. A., et al., 2015. Increased stability of heterogeneous ribonucleoproteins by a deacetylase inhibitor. Biochim. Biophys. Acta. 1849, 1095-103.

Kurtin, S. E., Bilotti, E., 2013. Novel agents for the treatment of multiple myeloma: proteasome inhibitors and immunomodulatory agents. J. Adv. Pract. Oncol. 4, 307-21.

Laguesse, S., et al., 2015. A Dynamic Unfolded Protein Response Contributes to the Control of Cortical Neurogenesis. Dev. Cell. 35, 553-67.

Lamothe, B., et al., 2015. Proteasome inhibitor carfilzomib complements ibrutinib's action in chronic lymphocytic leukemia. Blood. 125, 407-10. 
Lee, G., et al., 2009. Modelling pathogenesis and treatment of familial dysautonomia using patient-specific iPSCs. Nature. 461, 402-6.

Lefler, S., et al., 2015. Familial Dysautonomia (FD) Human Embryonic Stem Cell Derived PNS Neurons Reveal that Synaptic Vesicular and Neuronal Transport Genes Are Directly or Indirectly Affected by IKBKAP Downregulation. PLoS One. 10, e0138807.

Livak, K. J., Schmittgen, T. D., 2001. Analysis of relative gene expression data using realtime quantitative PCR and the 2(-Delta Delta C(T)) Method. Methods. 25, 402-8.

Mackay-Sim, A., 2012. Concise review: Patient-derived olfactory stem cells: new models for brain diseases. Stem Cells. 30, 2361-5.

Marchetto, M. C., et al., 2011. Induced pluripotent stem cells (iPSCs) and neurological disease modeling: progress and promises. Hum. Mol. Genet. 20, R109-15.

Mimura, N., et al., 2014. Selective and potent Akt inhibition triggers anti-myeloma activities and enhances fatal endoplasmic reticulum stress induced by proteasome inhibition. Cancer Res. 74, 4458-69.

Mitsiades, C. S., 2015. Therapeutic landscape of carfilzomib and other modulators of the ubiquitin-proteasome pathway. J. Clin. Oncol. 33, 782-5.

Modernelli, A., et al., 2015. EGCG antagonizes Bortezomib cytotoxicity in prostate cancer cells by an autophagic mechanism. Sci. Rep. 5, 15270.

Mor, E., et al., 2013. MicroRNA-382 expression is elevated in the olfactory neuroepithelium of schizophrenia patients. Neurobiol. Dis. 55, 1-10.

Morini, E., et al., 2016. Sensory and autonomic deficits in a new humanized mouse model of familial dysautonomia. Hum. Mol. Genet. 25, 1116-28. 
Moulton, V. R., et al., 2014. Ubiquitination regulates expression of the serine/arginine-rich splicing factor 1 (SRSF1) in normal and systemic lupus erythematosus (SLE) T cells. J. Biol. Chem. 289, 4126-34.

Muchtar, E., et al., 2016. A practical review on carfilzomib in multiple myeloma. Eur. J. Haematol. 96, 564-77.

Naftelberg, S., et al., 2016. Phosphatidylserine Ameliorates Neurodegenerative Symptoms and Enhances Axonal Transport in a Mouse Model of Familial Dysautonomia. PLoS Genet. 12, e1006486.

Nguyen, L. S., et al., 2016. Profiling olfactory stem cells from living patients identifies miRNAs relevant for autism pathophysiology. Mol. Autism. 7, 1.

Norcliffe-Kaufmann, L., et al., 2016. Familial Dysautonomia: History, Genotype, Phenotype and Translational Research. Prog. Neurobiol.

Ravid, T., Hochstrasser, M., 2008. Diversity of degradation signals in the ubiquitinproteasome system. Nat. Rev. Mol. Cell. Biol. 9, 679-90.

Sado, M., et al., 2009. Protective effect against Parkinson's disease-related insults through the activation of XBP1. Brain Res. 1257, 16-24.

Sardana, D., et al., 2011. Drug repositioning for orphan diseases. Brief Bioinform. 12, 346-56.

Schratt, G., 2009. microRNAs at the synapse. Nat. Rev. Neurosci. 10, 842-9.

Scott, K., et al., 2016. Bortezomib for the treatment of multiple myeloma. Cochrane Database Syst. Rev. 4, CD010816.

Slaugenhaupt, S. A., et al., 2001. Tissue-specific expression of a splicing mutation in the IKBKAP gene causes familial dysautonomia. Am. J. Hum. Genet. 68, 598-605.

Slaugenhaupt, S. A., et al., 2004. Rescue of a human mRNA splicing defect by the plant cytokinin kinetin. Hum. Mol. Genet. 13, 429-36. 
Svejstrup, J. Q., 2007. Elongator complex: how many roles does it play? Curr. Opin. Cell Biol. 19, 331-6.

Tsimokha, A. S., et al., 2014. DNA damage modulates interactions between microRNAs and the $26 \mathrm{~S}$ proteasome. Oncotarget. 5, 3555-67.

Ueki, Y., et al., 2016. Loss of Ikbkap Causes Slow, Progressive Retinal Degeneration in a Mouse Model of Familial Dysautonomia. eNeuro. 3.

Vlachos, I. S., et al., 2015a. DIANA-TarBase v7.0: indexing more than half a million experimentally supported miRNA:mRNA interactions. Nucleic Acids Res. 43, D1539.

Vlachos, I. S., et al., 2015b. DIANA-miRPath v3.0: deciphering microRNA function with experimental support. Nucleic Acids Res. 43, W460-6.

Volvert, M. L., et al., 2012. MicroRNAs tune cerebral cortical neurogenesis. Cell Death Differ. 19, 1573-81.

$\mathrm{Xu}, \mathrm{H}$., et al., 2015. Dimerization of elongator protein 1 is essential for Elongator complex assembly. Proc. Natl. Acad. Sci. U. S. A. 112, 10697-702.

Yoshida, M., et al., 2015. Rectifier of aberrant mRNA splicing recovers tRNA modification in familial dysautonomia. Proc. Natl. Acad. Sci. U. S. A. 112, 2764-9.

Zeltner, N., et al., 2016. Capturing the biology of disease severity in a PSC-based model of familial dysautonomia. Nat. Med. 22, 1421-1427.

Zhang, X., et al., 2015. MicroRNA-101 Suppresses Tumor Cell Proliferation by Acting as an Endogenous Proteasome Inhibitor via Targeting the Proteasome Assembly Factor POMP. Mol. Cell. 59, 243-57. 


\section{FIGURE LEGENDS}

Figure 1. Modulation of IKBKAP alternative splicing and IKAP/hELP1 protein expression level by bortezomib alone or combined to kinetin.

Increasing doses of bortezomib $(1,5,10,15,20,25,30,60,125,250,500,1000 \mathrm{nM})$ were applied on C1 and C3 hOE-MSCs (Control, filled circles) as well as FD2 and FD4 hOEMSCs (FD, empty circles) over a $48 \mathrm{~h}$ time period and cell viability was assessed by crystal violet assay (A). The WT:MU ratio of IKBKAP transcripts from FD4 hOE-MSCs treated with 5-30 nM bortezomib was assessed by RT-qPCR (B). IKAP/hELP1 expression profile was visualized by western blot for the same bortezomib concentrations (C). Quantity of sample loading was normalized by $\beta$-actin. In combination experiments, FD4 hOE-MSCs were treated over $48 \mathrm{~h}$ with $10 \mathrm{nM}$ bortezomib (BTZ), or $25 \mu \mathrm{M}$ kinetin $(\mathrm{K})$, or both drugs (BTZ + K). The WT:MU ratio of IKBKAP transcripts was determined by RT-qPCR (D), whereas IKAP/hELP1 protein level was estimated by western immunoblotting technique (E). Arrows indicate the bortezomib concentration used for synergic effect experiment, whereas error bars indicate standard error for two independent experiments (A, B, D).

Figure 2. Enhanced effect of epoxomicin and kinetin on IKBKAP mRNA and IKAP/hELP1 profile.

Increasing doses of epoxomicin $(1,5,10,15,20,25,30,60,125,250,500,1000 \mathrm{nM})$ were applied on $\mathrm{C} 1$ and $\mathrm{C} 3$ hOE-MSCs (Control, filled circles) as well as FD2 and FD4 hOEMSCs (FD, empty circles) over a $48 \mathrm{~h}$ time period and cell viability was assessed by crystal violet assay (A). The WT:MU ratio of IKBKAP transcripts from FD4 hOE-MSCs treated with 1-30 nM epoxomicin was assessed by RT-qPCR (B). IKAP/hELP1 expression profile was visualized by western blot for the same epoxomicin concentrations (C). In combination 
experiments, FD4 hOE-MSCs were treated over 48h with $15 \mathrm{nM}$ epoxomicin (EPX), or 25 $\mu \mathrm{M}$ kinetin $(\mathrm{K})$, or both drugs $(\mathrm{EPX}+\mathrm{K})$. The WT:MU ratio of IKBKAP splicing isoforms was determined by RT-qPCR (D), whereas IKAP/hELP1 protein level was estimated by western immunoblotting technique (E). Arrows indicate the epoxomicin concentration used for synergic effect experiment (A, B). Error bars indicate standard error for two independent experiments (A, B, D).

Figure 3. Strong effects of carfilzomib on IKBKAP mRNA and IKAP/hELP1 protein profiles.

Increasing doses of carfilzomib $(1,5,10,15,20,25,30,60,125,250,500,1000 \mathrm{nM})$ were applied on $\mathrm{C} 1$ and $\mathrm{C} 3$ hOE-MSCs (Control, filled circles) as well as FD2 and FD4 hOEMSCs (FD, empty circles) over a 48h time period and cell viability was assessed by crystal violet assay (A). The WT:MU ratio of IKBKAP transcripts from FD4 hOE-MSCs treated with 5-125 nM carfilzomib was assessed by RT-qPCR (B). IKAP/hELP1 expression profile was visualized by western blot for the same carfilzomib concentrations (C). Quantity of sample loading was normalized by $\beta$-actin. In combination experiments, FD4 hOE-MSCs were treated over $48 \mathrm{~h}$ with $30 \mathrm{nM}$ carfilzomib (CFZ), or $25 \mu \mathrm{M}$ kinetin $(\mathrm{K})$, or both drugs $(\mathrm{CFZ}+$ $\mathrm{K})$. The WT:MU ratio of IKBKAP isoforms was determined by RT-qPCR (D), whereas IKAP/hELP1 protein level was estimated by western immunoblotting technique (E). Arrows indicate the carfilzomib concentration used for synergic effect experiment, whereas error bars indicate standard error for two independent experiments (A, B, D).

\section{Figure 4. Overactivity of $26 \mathrm{~S}$ proteasome in $\mathrm{FD}$}

$26 \mathrm{~S}$ proteasome activity was assessed in two control (C1 and C3) and two FD (FD2 and FD4) hOE-MSC cultures by measuring chymotrypsin-like activity using a fluorescent assay. 
Enzymatic activity of each culture is indicated as the fluorescence signal (RFU) emitted by 30000 cells. Error bars indicate standard error. Asterisks indicate significant difference between the groups $(* *, P<0.01)$. 


\section{AUTHOR CONTRIBUTIONS}

E.C.I. designed and obtained funding for the study. M.H. and E.C.I performed the experiments. M.H. and E.C.I. analyzed the data and wrote the manuscript. 


\section{COMPETING INTERESTS}

The authors declare that they have no competing interests. 

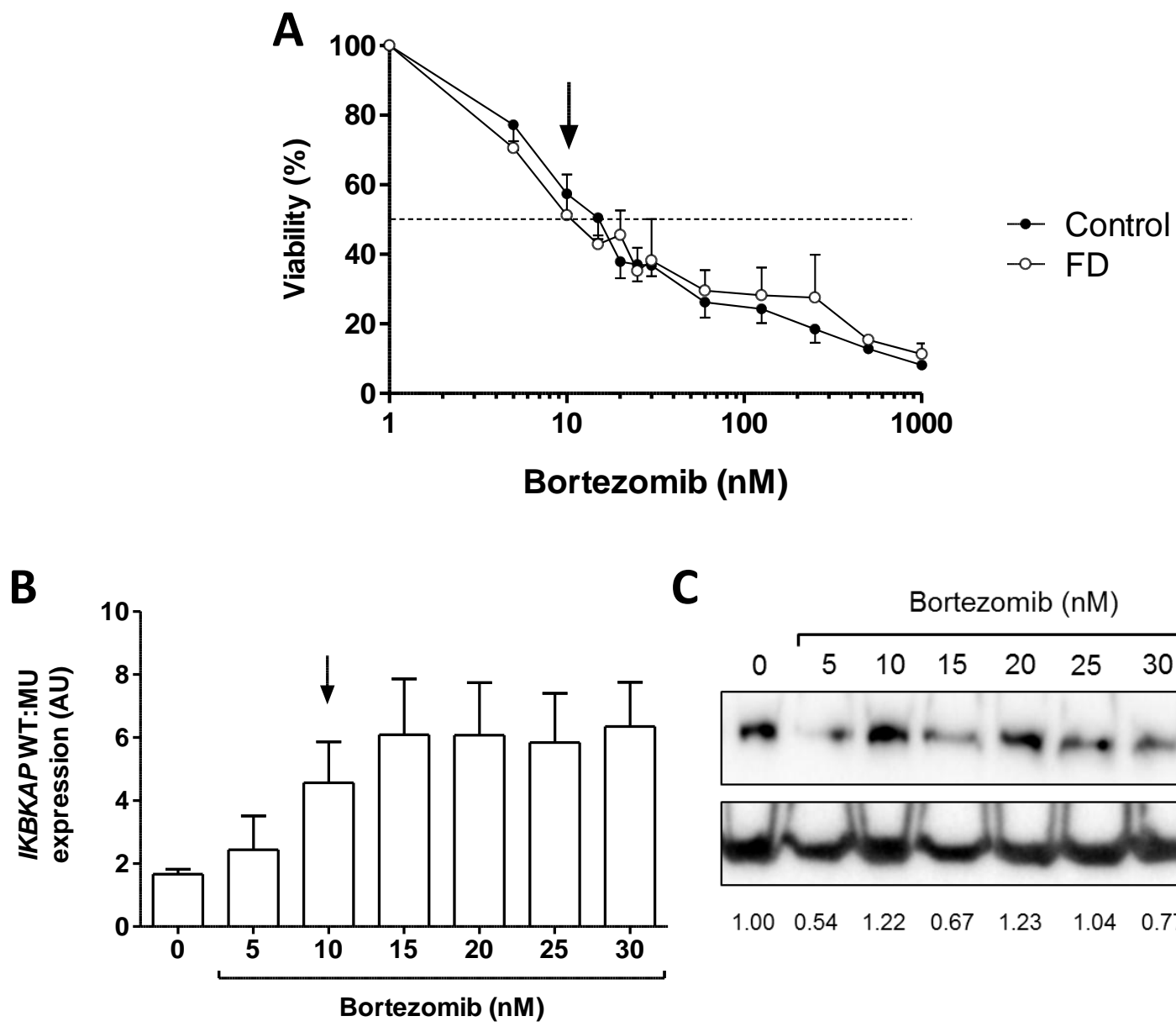

C Bortezomib (nM)

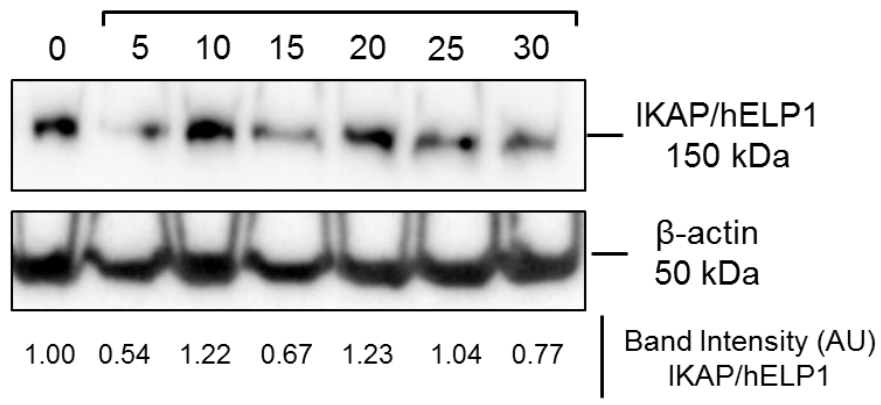

D

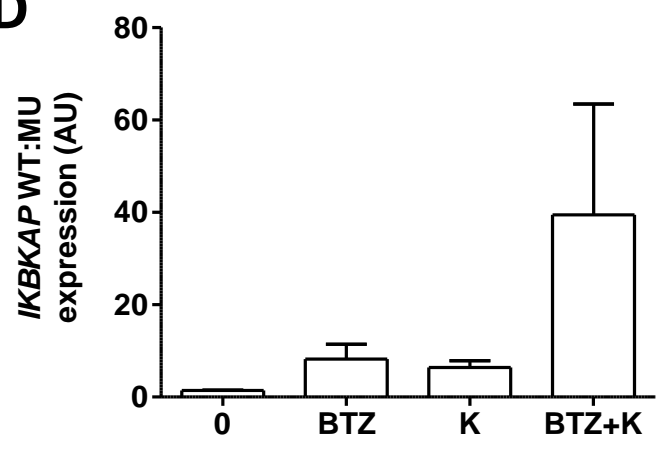

E

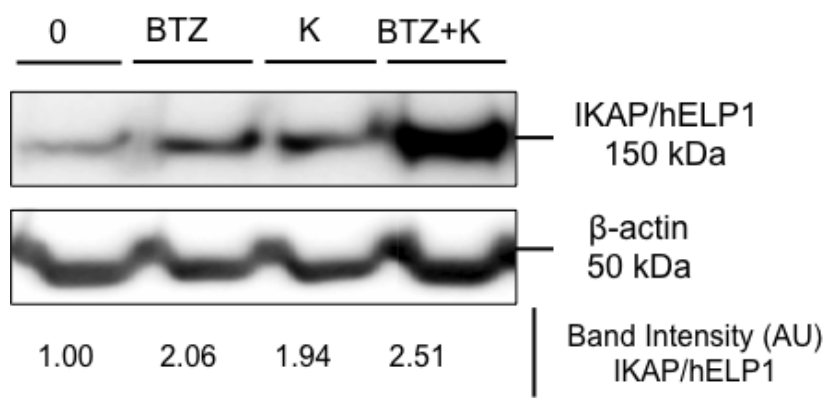



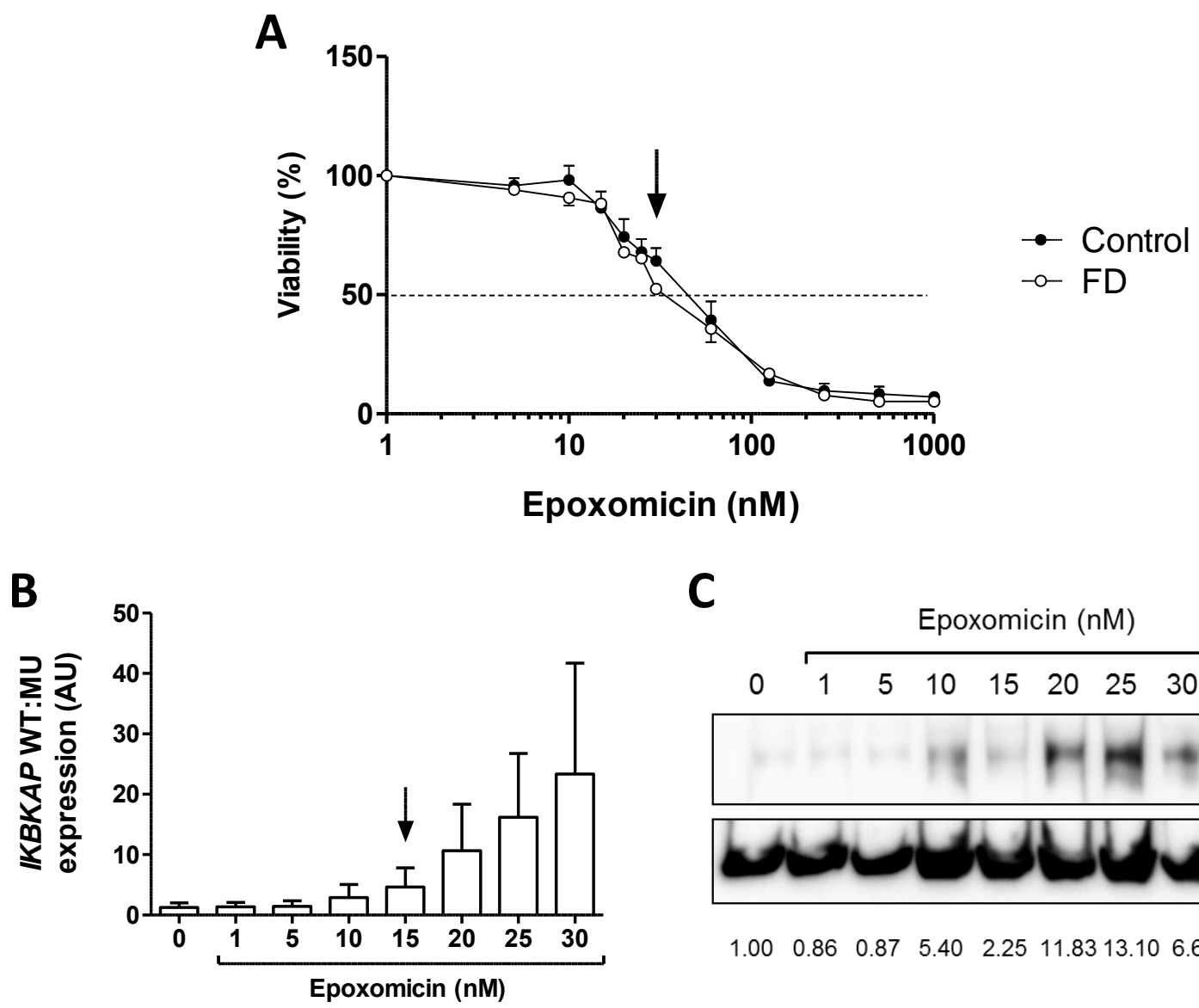

C

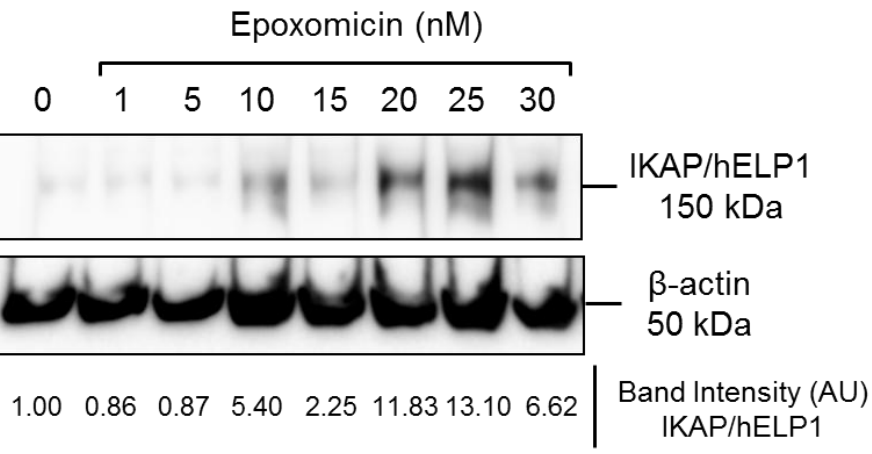

D

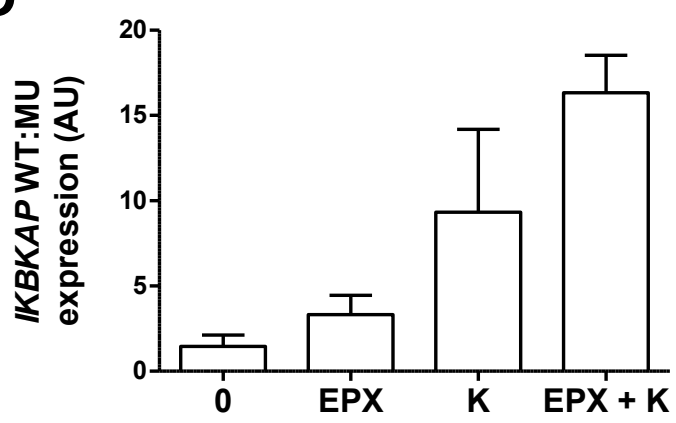

E

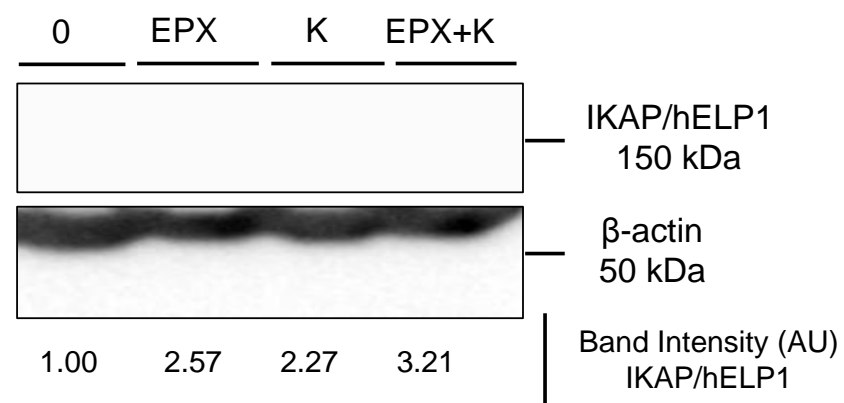

Figure 2 


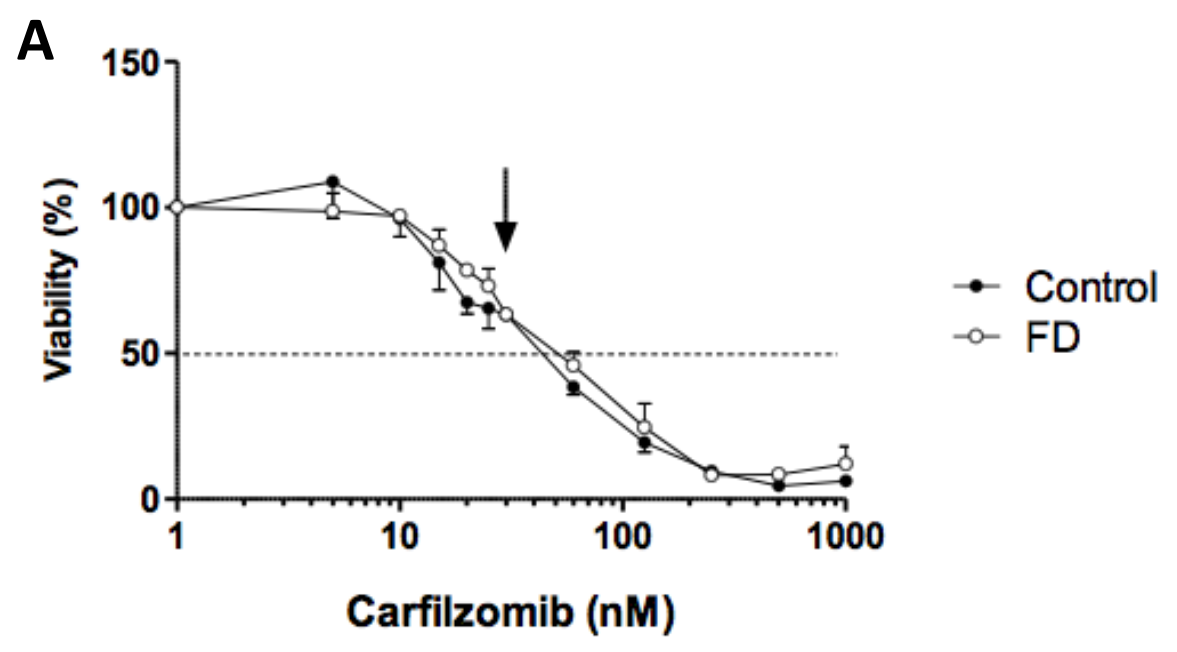

B

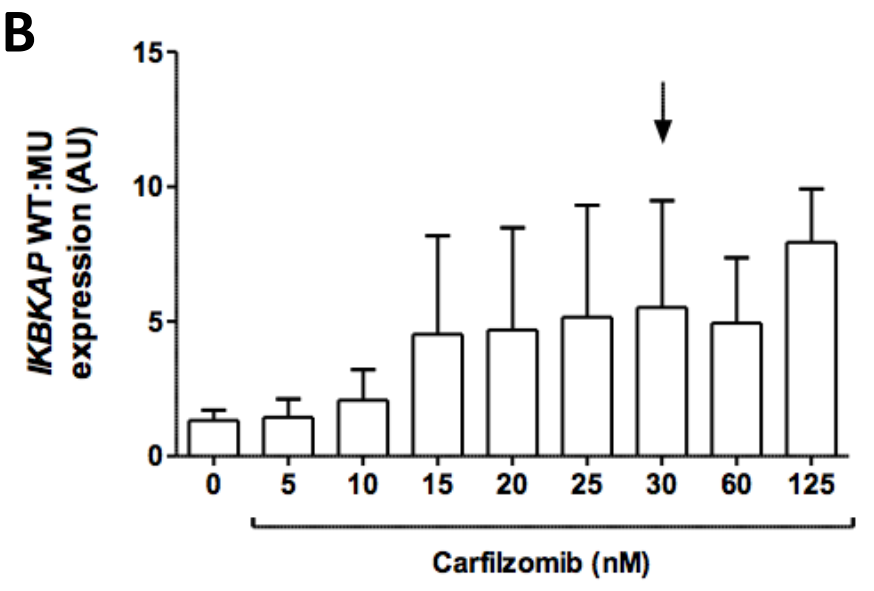

D

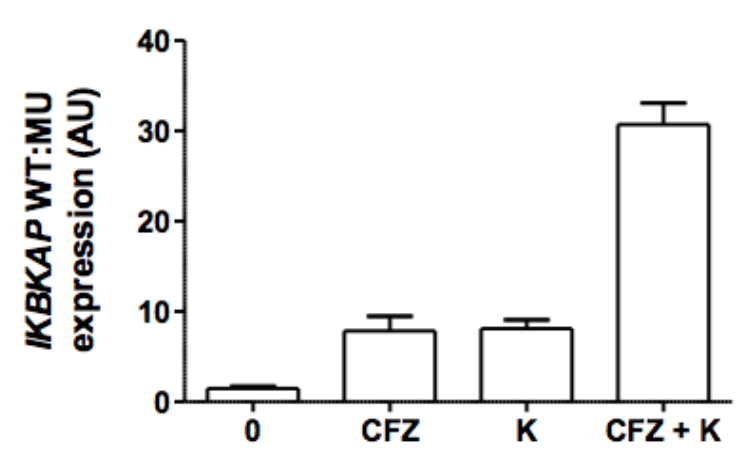

C
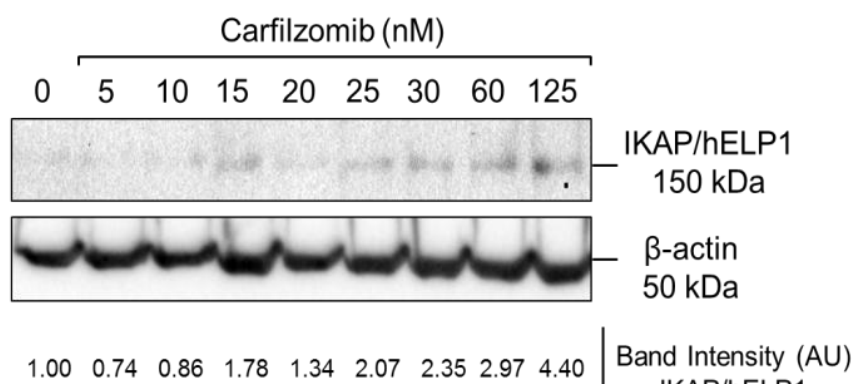
IKAP/hELP1

E

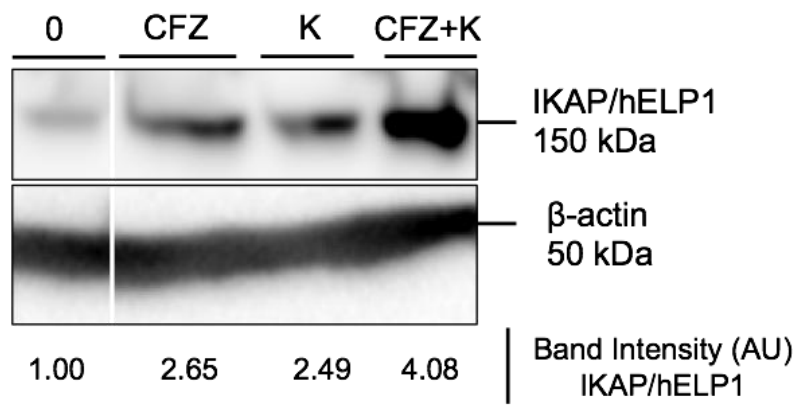

Figure 3 
Table 1 Dysregulated miRNAs in control and FD hOE-MSCs induced by carfilzomib treatment

\begin{tabular}{lrl} 
miRNA & FC & P-value \\
\hline CTRL + CFZ vs. CTRL & \\
\hline miR-126-3p & 1.57 & $2.91 \mathrm{E}-02$ \\
miR-425-5p & 1.50 & $4.94 \mathrm{E}-02$ \\
miR-636 & 1.48 & $4.14 \mathrm{E}-02$ \\
miR-23b-3p & 0.75 & $5.09 \mathrm{E}-03$ \\
miR-493-3p & 0.73 & $1.30 \mathrm{E}-03$ \\
\hline \multicolumn{3}{c}{ FD + CFZ vs. FD } \\
\hline miR-126-3p & 2.42 & $9.03 \mathrm{E}-04$ \\
miR-376a-3p & 2.02 & $9.38 \mathrm{E}-03$ \\
miR-365a-3p & 1.41 & $3.57 \mathrm{E}-02$ \\
miR-28-5p & 0.77 & $2.22 \mathrm{E}-02$ \\
miR-532-5p & 0.74 & $6.74 \mathrm{E}-03$ \\
miR-137 & 0.74 & $1.01 \mathrm{E}-02$ \\
let-7c-5p & 0.70 & $3.75 \mathrm{E}-02$ \\
miR-92a-3p & 0.69 & $4.45 \mathrm{E}-02$ \\
miR-636 & 0.69 & $3.42 \mathrm{E}-02$ \\
miR-660-5p & 0.69 & $1.66 \mathrm{E}-02$ \\
miR-210-3p & 0.68 & $4.76 \mathrm{E}-02$ \\
miR-339-5p & 0.67 & $4.61 \mathrm{E}-02$ \\
miR-422a & 0.66 & $1.52 \mathrm{E}-02$ \\
miR-342-3p & 0.65 & $1.85 \mathrm{E}-02$ \\
\hline
\end{tabular}

Significantly dysregulated miRNAs following $24 \mathrm{~h}$ application of $125 \mathrm{nM}$ calfilzomib (CFZ) on four control hOE-MSCs (CTRL) and four FD hOE-MSCs are indicated. Mean FC threshold was set at 1.3 and significance was assessed using paired mode of the parametric Student's t-test with p-value threshold set at 0.05 . 
Table 2 Validated mRNA targets of dysregulated miRNAs associated with the ubiquitin/proteasome machinery

\begin{tabular}{|c|c|c|c|c|c|c|c|}
\hline Target & miRNA & Target & miRNA & Target & miRNA & Target & miRNA \\
\hline BIRC6 & $\mathrm{H}$ & PSMD8 & $\mathrm{C}$ & UBE2Q2 & $\mathrm{F}, \mathrm{H}, \mathrm{N}$ & $\overline{U S P 21}$ & $\mathrm{E}$ \\
\hline$C D C 34$ & $E$ & PSME1 & B & UBE2R2 & $\mathrm{B}, \mathrm{N}$ & USP22 & $\mathrm{C}, \mathrm{H}, \mathrm{J}, \mathrm{M}$ \\
\hline$C Y L D$ & $\mathrm{I}, \mathrm{L}$ & PSME3 & $\mathrm{J}, \mathrm{N}$ & UBE2V2 & B & USP24 & $\mathrm{E}$ \\
\hline ITCH & $\mathrm{L}$ & PSME4 & G & UBE2W & $\mathrm{E}, \mathrm{N}$ & USP28 & $B, F, J, N$ \\
\hline MOCS3 & $E, I$ & PSMF1 & $N$ & UBE2Z & $\mathrm{F}, \mathrm{H}, \mathrm{M}, \mathrm{N}$ & USP3 & K \\
\hline PSMA2 & $E$ & PSMG2 & $E$ & UBE3C & $\mathrm{D}, \mathrm{F}, \mathrm{L}$ & USP30 & G \\
\hline PSMA3 & K & PSMG3 & $\mathrm{N}$ & $U B E 4 B$ & $E, I, N$ & USP31 & $B, C, N$ \\
\hline PSMA4 & $\mathrm{N}$ & SAE1 & $\mathrm{C}, \mathrm{J}$ & UBQLN1 & $\mathrm{C}, \mathrm{E}, \mathrm{G}, \mathrm{I}$ & USP32 & $\mathrm{E}$ \\
\hline PSMA7 & $\mathrm{N}$ & SMURF1 & $C$ & UBQLN2 & C & USP33 & $\mathrm{N}$ \\
\hline PSMB1 & $\mathrm{N}$ & TRAF7 & $A$ & UBR1 & $\mathrm{E}, \mathrm{N}$ & USP36 & $\mathrm{F}, \mathrm{N}$ \\
\hline PSMB2 & $E$ & UBA1 & G & UBR2 & $\mathrm{C}, \mathrm{E}$ & USP37 & $\mathrm{B}, \mathrm{L}$ \\
\hline PSMB3 & $E, I$ & UBA6 & $\mathrm{H}, \mathrm{M}$ & UBR3 & B & USP38 & $E, J, N$ \\
\hline PSMB6 & $F, L, N$ & $U B B$ & $B, J, L$ & UBR4 & $B, D, H$ & USP42 & $\mathrm{E}$ \\
\hline PSMC1 & $\mathrm{F}$ & $U B C$ & $\mathrm{~L}$ & UBR5 & $B, C$ & USP45 & $\mathrm{N}$ \\
\hline PSMC3 & $\mathrm{N}$ & UBE2D1 & B & UBXN4 & $\mathrm{F}, \mathrm{N}$ & USP46 & $B, F$ \\
\hline PSMC5 & $\mathrm{H}$ & UBE2D2 & 1 & UBXN7 & I & USP47 & $\mathrm{E}$ \\
\hline PSMD1 & C & UBE2D3 & $B, D, E, G, L, N$ & UCHL1 & $\mathrm{G}, \mathrm{I}, \mathrm{N}$ & USP48 & $A$ \\
\hline PSMD11 & $\mathrm{C}, \mathrm{E}, \mathrm{N}$ & UBE2E2 & B & UCHL3 & $\mathrm{L}$ & USP49 & $B, I$ \\
\hline PSMD12 & $\mathrm{C}, \mathrm{N}$ & UBE2G1 & $\mathrm{N}$ & UCHL5 & $\mathrm{N}$ & USP5 & $B, C, I$ \\
\hline PSMD14 & B & $U B E 2 G 2$ & $C, E$ & USP1 & $E, H, J, N$ & USP51 & 1 \\
\hline PSMD2 & $\mathrm{N}$ & $U B E 2 H$ & $\mathrm{H}, \mathrm{I}, \mathrm{N}$ & USP10 & $\mathrm{G}, \mathrm{N}$ & USP53 & B \\
\hline PSMD3 & $\mathrm{I}, \mathrm{N}$ & UBE2J1 & $\mathrm{E}$ & USP11 & $\mathrm{N}$ & USP9X & $B, D, E, H, I, L, M, N$ \\
\hline PSMD5 & $\mathrm{N}$ & UBE2K & $A, E, M, N$ & USP13 & $\mathrm{J}, \mathrm{N}$ & WWP2 & B \\
\hline PSMD6 & C & UBE2M & B & USP14 & $\mathrm{J}$ & & \\
\hline PSMD7 & D & UBE2N & $\mathrm{J}, \mathrm{L}$ & USP19 & $\mathrm{J}$ & & \\
\hline Target & miRNA & Target & miRNA & Target & miRNA & Target & miRNA \\
\hline$I T C H$ & $\mathrm{O}, \mathrm{Q}$ & PSMD8 & $\mathrm{Q}$ & $\overline{U B E 3 A}$ & $\mathrm{P}$ & USP34 & $\mathrm{P}$ \\
\hline PSMA3 & $\mathrm{Q}$ & SAE1 & Q & UBE3B & $Q$ & USP36 & $\mathrm{F}, \mathrm{S}$ \\
\hline PSMA4 & $\mathrm{R}$ & SMURF2 & $P$ & UBE3C & $\mathrm{F}$ & USP46 & $\mathrm{F}$ \\
\hline PSMB6 & $\mathrm{F}$ & TRAF7 & $A$ & $U B E 4 B$ & $\mathrm{P}$ & USP47 & $\mathrm{P}$ \\
\hline PSMB8 & Q & $U B C$ & Q & UBQLN4 & $\mathrm{R}$ & USP48 & $\mathrm{A}, \mathrm{O}$ \\
\hline PSMC1 & $\mathrm{F}$ & UBE2D3 & $\mathrm{S}$ & UBR4 & $\mathrm{R}$ & USP49 & $\mathrm{Q}$ \\
\hline PSMD14 & $\mathrm{R}$ & UBE2E1 & $\mathrm{S}$ & UBR5 & $\mathrm{R}$ & USP54 & 0 \\
\hline PSMD2 & $Q$ & UBE2K & $A, P, S$ & $U B X N 2 A$ & $\mathrm{P}$ & USP9X & $\mathrm{P}$ \\
\hline PSMD3 & 0 & UBE2Q2 & $\mathrm{F}$ & UBXN4 & $\mathrm{F}$ & WWP2 & Q \\
\hline PSMD6 & $Q$ & $U B E 2 R 2$ & $S$ & USP22 & $P$ & & \\
\hline PSMD7 & $\mathrm{P}$ & UBE2Z & $F, Q$ & USP28 & $\mathrm{F}$ & & \\
\hline
\end{tabular}

Validated mRNA targets of the carfilzomib-induced dysregulated miRNAs (Table A) and of dysregulated miRNAs in FD pathology (Table B) were obtained using the Tarbase v7.0, miRTarBase and miRWalk2.0 databases. Targets were compared to a list of 190 ubiquitin/proteasome-related genes (Additional File 2). Each letter in the 'miRNA' column refers to a dysregulated miRNA as follows: A, miR-126-3p; B, miR-23b-3p; C, miR-425-5p; 
D, miR-493-3p; E, let-7c-5p; F, miR-137; G, miR-210-3p; H, miR-28-5p; I, miR-342-3p; J, miR-365a-3p; K, miR-422a; L, miR-532-5p; M, miR-660-5p; N, miR-92a-3p. Letters in black refer to Tarbase v7.0 only, in brown to miRTarbase only, in blue to miRTarbase and miRWalk2.0, in purple to Tarbase v7.0 and miRTarbase, and in green to all three databases. 
Supplementary Material
Click here to download Supplementary Material: Additional_Files_230317.docx

Click here to download Supplementary Material: Additional_Files_230317.docx

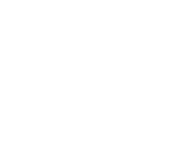

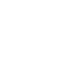

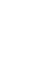
$\sqrt{3}$ (1) (1) (1) (1)

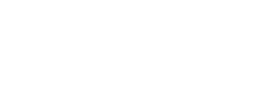

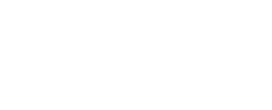
$+2$

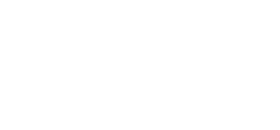
, . . . . . . . . . . . . . . . . . . . - 